RESEARCH PAPER

\title{
ASSESSING THE SUITABILITY OF WOVEN FABRIC AND COMPOSITE TEXTILE TECHNIQUES FOR MURAL PRODUCTION
}

\author{
E. K. Howard ${ }^{1}$ and N. A. Opoku-Asare ${ }^{2}$ \\ ${ }^{1}$ Department of Industrial Art, KNUST, Kumasi \\ ${ }^{2}$ Department of General Art Studies, KNUST, Kumasi
}

\begin{abstract}
:
This art studio experimental study explored the suitability of woven cotton fabric as alternative material for creating pictorial designs for murals based on the batik, tie-and-dye, screen printing, appliqué and embroidery techniques in textiles. While painted and sculpted murals abound in Ghana, the study found textile murals a seemingly unknown art form. The experiments proved that plain woven mercerized cotton and damask fabrics are good materials for creating pictorial designs for textile murals. With the exception of the tie-dye technique which generates undefined pictorial designs, the batik, screen printing, appliqué and embroidery techniques yield significantly well-defined designs for producing textile murals. Displaying the textile murals in dry, airy places prevents fungal attack and easy deterioration which ensures a long shelf life for the murals.
\end{abstract}

Keywords: Cotton; fabric; textiles technique; pictorial design; mural.

\section{INTRODUCTION}

A mural is an extremely large work of art, most often a painting applied to the surface of a wall, ceiling, or floor for aesthetic and didactic purposes (Wisegeek, 2012). They may be executed on wooden panels and fixed on walls or painted on canvas in the artist's studio and glued to walls or ceilings, interiors and exteriors of buildings. Adams (1999) refers to murals as fresco and explains that a mural can be applied to and made integral part of the surface of a wall, ceiling or floor. Tarantino (2011) explains that any tile or fired clay that is glazed with a design or a number of such tiles that are individual segments of a larger design and fixed to a wall or floor can be referred to as a mural.

Murals have existed since prehistoric times. Typical examples are those discovered in 1879 in the caves of Altamira (Northern Spain). Ancient Egyptians decorated the walls of their palaces and tombs with brightly painted murals. While some of the Egyptian mural paintings depicted everyday life and life after death, Scholastic (2012) indicates that many told stories of gods, goddesses, and rulers. Greek and Roman murals depict scenic mountaintop, gardens, buildings, gods and heroes with the greatest murals being made during the Renaissance Period (Wisegreek, 2012). According to En- 


\section{Howard and Opoku-Asare}

carta (2009) and Demoli (2011), murals of the Early Christian and Byzantine periods show Biblical themes covering entire surfaces of walls and ceilings. Notable among them are those in the St. Peter's Basilica, Gothic churches and cathedrals in Rome; and Buddhist murals in rock-cut shrines at Ajanta, India.

A major feature of murals is their large sizes. Mural design subjects are made in large dimensions to particular aesthetic limitations and functions (Bhuyan, 2011). Characteristically, a mural is organically connected to the architectural scheme of the building it decorates. A particularly distinguishing characteristic of mural painting (Campbell, 2003) is that the architectural elements of the given space are harmoniously incorporated into the picture. Anaba (1995) explains that the selection of colour, design and production technique goes hand in hand to enhance the beauty and alter the sensation of spatial proportion of the building. He further adds that murals are the only forms of pictorial designs that are truly three dimensional. They make space appear more confining and alter the viewer's perception by creating an illusion of depth in walls, ceilings and floors. Murals may be made in silhouette, realistic or abstract forms with harmonious colours to create transparency, solidity, depth or perspective.

Significantly, murals are mostly made for aesthetic purposes but they also serve emotional and psychological purposes. Mural designs (Encarta, 2009) are made to reflect social, religious, patriotic activities and the culture of a people. Donitz (2004) observes that themes based on civil rights and social justice including life and death, youth, education, relationships, and cultural identity are portrayed in murals; but religious matters are the most influential and common. To Donitz, murals are very important tools for social and political expression and can be used to gauge the concerns and hopes of a community and to inspire and foster a sense of identity and pride in one's culture. They are powerful forms of social communica- tion in that they are accessible to everyone. They communicate direct and tangible messages to the common people in their neighbourhood and therefore reach larger audiences than museums and galleries. As instructional materials, murals facilitate teaching and learning of aesthetics, appreciation and criticism. Mural making in school affords mutual interactions between teachers and students, and also among students. Osumi (2000) therefore encourages teachers to think of murals as an interactive process that blends collaborative grouping, history, current events, local community, social change, leadership skills, and creative development.

Mural techniques include encaustic painting, fresco, oil painting and tempera (Asihene, 2004; Encarta, 2009). Some modern murals are composed of photographs and mosaics. Although fresco is the most commonly used technique, sometimes artists paint on canvas and fix it onto a wall surface using the Marouflage technique (The World Book Encyclopaedia, 2000). The Japanese and Chinese are noted for making murals based on hand colour print, lacquer-print and wood-cut or block printing methods on silk (Howard, 2006). Murals based on fabric and textile techniques seem to have limited application probably because of their susceptibility to deterioration with age. Since creativity through experimentation underlies innovation, textile murals can serve as a hatchery for new ideas in studio art production (Howard, 2006).

\section{MATERIALS AND METHODS}

The most significant material used for the experimental textile murals is fabric which is generally used for the production of garments, fashionable articles, furnishing and upholstery. In this project, mercerized cotton and damask fabrics in plain weave were found most appropriate because they have no design, are very absorbent, relatively strong and offer a good ground for dyeing and printing. Other materials used were PVA glue (carpenter's or white glue), printing paste, assorted vat dyes, paraffin 
wax, organdie mesh, sodium hydroxide, sodium hydrosulphite, different types of papers, opaque ink, potassium dichromate and wood (Wawa board and Ceiba plywood). These items were used in combinations in different ways to suit the materials and techniques employed for the murals.

The experiments which sought to assess the suitability of mercerized cotton and damask fabrics for creating murals were conducted in the dyeing and printing studios of the Textiles Section of the Faculty of Art, Kwame Nkrumah University of Science and Technology. The only tools and equipment available to the researchers in these art studios for the experimental murals included printing table, developing table, light box, electric sewing machine, embroidery machine, gas cooker, pressing iron and ironing board. The study focused on finding out the following:

1. rate of absorption of mercerized cotton and damask fabrics;

2. stability of mercerized cotton and damask fabrics for the batik and tie-dye processes;

3. adhesion power of Polyvinyl alcohol (PVA) glue on mercerized cotton and damask fabrics;

4. wash fastness of vat dyes used on both fabrics;

5. affinity of mercerized cotton and damask fabrics for water-based print paste;

6. preservation of cotton fabric from moth or insect attack;

7. suitability of fabric as a medium for mural production.

\section{RESULTS AND DISCUSSION}

This section provides a discussion and analysis of the results of the experiments on the suitability of woven fabrics for mural production. It describes the results of the preliminary survey of local murals, the assessment of the selected woven fabrics and textile techniques adopted for the experimental murals, and the design and production processes adopted for the two sample textile murals.

\section{Survey of local murals}

The survey of libraries, hotels, buildings, and educational institutions in the Kumasi metropolis revealed the use of indoor and outdoor murals depicting Ghanaian traditional themes used symbolically to welcome guests and promote Ghanaian culture. Murals found portrayed academic themes such as teaching and learning, sports, entertainment and feasting activities. The public edifices have murals with religious, political, social, and commercial themes aimed at communication, advertisement and beautification. Biblical themes characterize church murals which serve as objects of worship and beautification. Murals in educational institutions and hotels are mostly paintings with few sculptural and ceramic forms.

The techniques used for the murals that were found during the survey are mainly fresco painting, slabbing, relief carving and modelling, which employ the use of brushes, chisels, modelling tools, oil paint, clay, cement, and wood. This apparently indicates that artists still employ conventional techniques, tools and materials for mural production. The absence of textile murals was clear from the survey, suggesting that the use of fabric and textile techniques has either not found application in mural production or textile murals are not widely available. This study therefore serves as a novelty that adds to and expands the scope of mural production in Ghana. Both textile artists and muralists can now explore this new concept to enhance their work for the artistic and economic development of Ghana.

\section{Assessment of woven fabrics for mural pro- duction}

The assessment of woven fabrics and selected textiles auxiliaries such as vat dyes, water based print paste, PVA glue and yarns was carried out under art studio conditions where conventional laboratory facilities for conducting chemical tests are non-existent. For this reason, the selected woven fabrics were subjected only to basic physical tests, including dyeing, printing, adhesion, cutting, washing, direct exposure 
143 Howard and Opoku-Asare

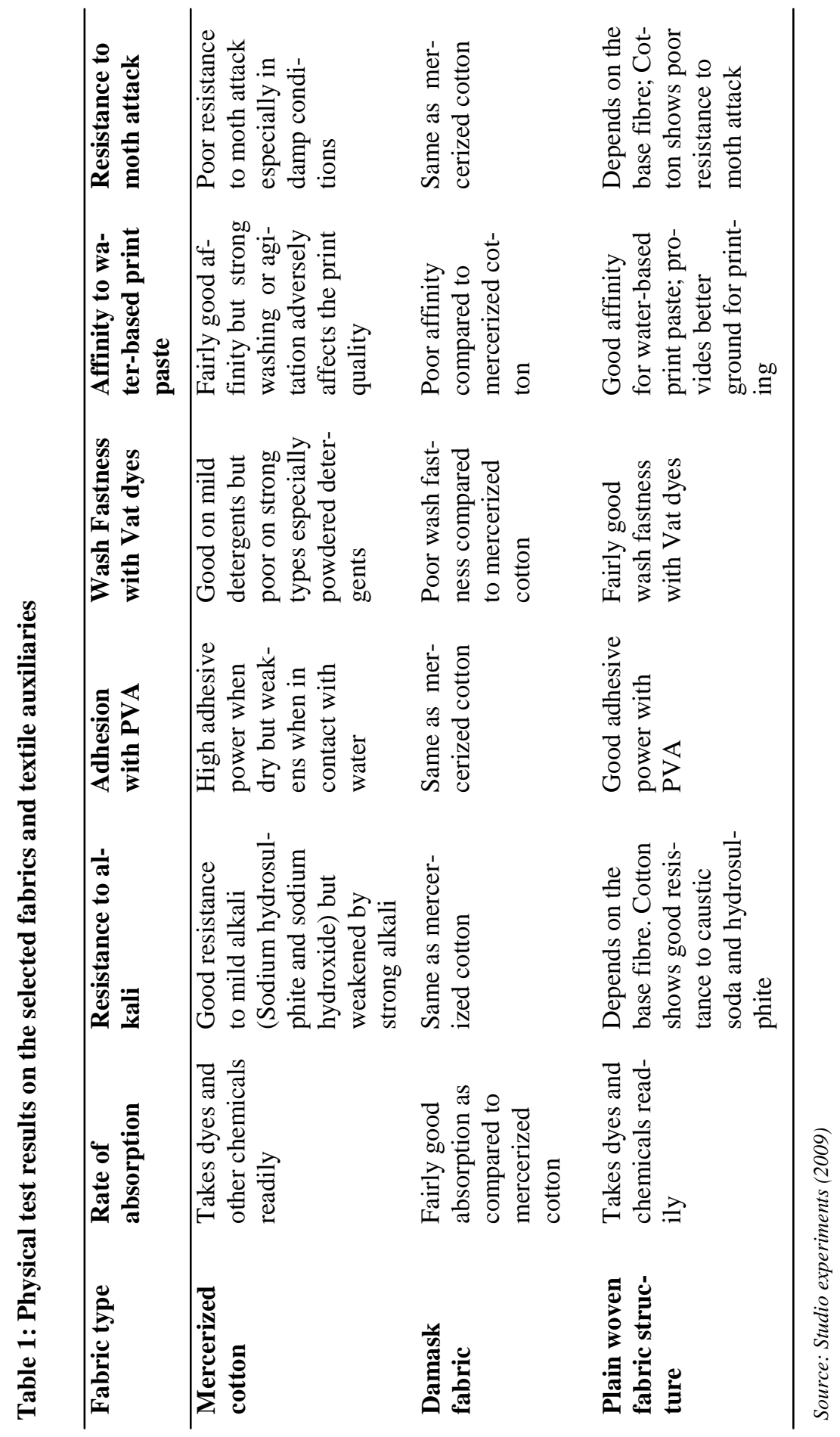

Journal of Science and Technology @ KNUST April 2012 
to sunlight, and prolonged contact with alkali solution, purposely to determine the rate of absorption, wash fastness, resistance to alkali and moth attack, the structural stability and tensile strength of the fabrics used. Table 1 shows the test results.

As indicated in Table 1, mercerized cotton and damask fabrics have good affinity for vat dyes and dye auxiliaries such as sodium hydrosulphite and sodium hydroxide. The vat dye, sodium hydrosulphite and sodium hydroxide used for the experiments was in the ratio of $1: 2: 2$ respectively for a 2-metre fabric. Both fabrics exhibit good adhesive power with PVA glue. They were both very absorbent as they take dyes and chemical finishes readily, have the ability to withstand weak alkali solution, fairly good wash fastness and offer a good ground for dyeing and printing. The fabrics in plain weave are dimensionally stable and strong due to equal distribution of warp and weft yarns along the length and width of the structures. However, excessive use of sodium hydroxide (caustic soda) weakens the cotton fabric and causes it to disintegrate thereby reducing the life span of the dyed fabric and hence the mural.

The implication of these tests is that care must be taken to use the right quantities of materials and chemicals in order to preserve the strength of the fabrics in order to prolong the shelf life of murals produced from them. These properties justify the suitability of plain mercerized cotton and damask fabrics and the selected textile auxiliaries for the production of textile murals. It is important to mention that batik, tiedye, screen printing, appliqué and embroidery were the main textile techniques employed for the study. The results of the experiments show the possibility of combining two or more textiles techniques for mural production.

One major limitation observed from the experiments is that the tie-and-dye technique is ineffective for producing figurative designs in fabrics because it cannot generate well-defined outlines in pictorial format. Batik, embroidery, appliqué, and screen printing are very effective but outstanding results could be obtained when two or more of the techniques are combined as was apparent in the sample textile murals. Printing on highly calendered damask fabric results in distorted design edges because the polished surface resists penetration of printing paste into the amorphous regions of the fibres. Mercerized cotton fabric gives little or no problem as the fabric takes up printing paste readily and gives sharp and accurate design edges.

Design and production of the textile murals Mural design is conceptualized in conformity with activities that are significant in the environment it is made to serve. In this project, both realistic and abstract themes were adopted to portray activities that are typically Ghanaian. Simplicity of forms and organisation of design elements are very essential factors in mural production. The designs were derived from imaginative composition, pictures in magazines and illustrations on sketch pads. These were modified to render them suitable to the tools, techniques and materials identified for the project. Several sketches of the mural designs were made in pencil on A4 size cartridge paper and two were selectively developed for production.

The following sections describe in detail the procedures and processes associated with the production of two textile murals which form the basis of this article.

\section{Mural 1: "The Pride of Ghanaian Women"}

The design is based on gorgeously clothed Ghanaian female figures drawn in semiabstracted form in a landscape picture plane. The design depicts a variety of textured fabrics in the background. Figure 1a represents Step 1 of the designing process and shows the outline drawing of the figures in pencil.

Step 2: Different textures were made on the fabrics covering the figures while the figures themselves were shaded flat in the form of silhouettes (Fig. 1b). 


\section{Howard and Opoku-Asare}

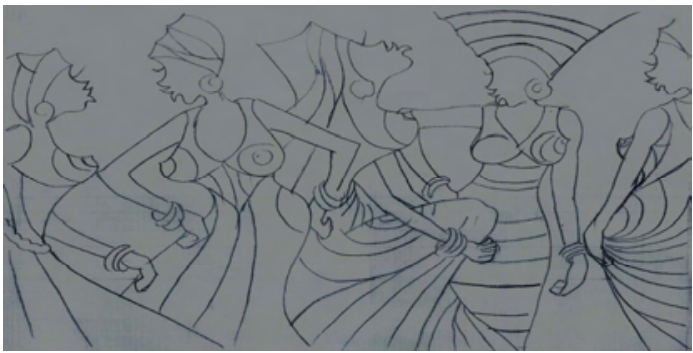

Fig. 1a: The outlined design

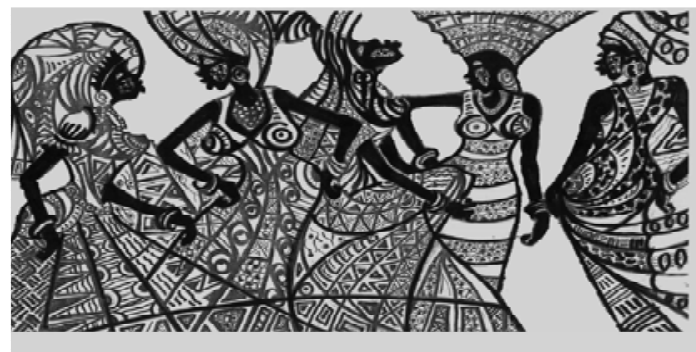

Fig. 1b: The female figures

Step 3: The background of the picture plane was covered with additional textures created with linear and geometric patterns of different sizes to give an impression of varied textured fabrics to create a form of bond between the figures and the background.

Step 4: After checking the proportions of the figures and rubbing off unwanted pencil marks, the design was inked with black Rotring ink to make the figures stand out as shown in Fig. 1c which also depicts the final design phase of Mural 1.

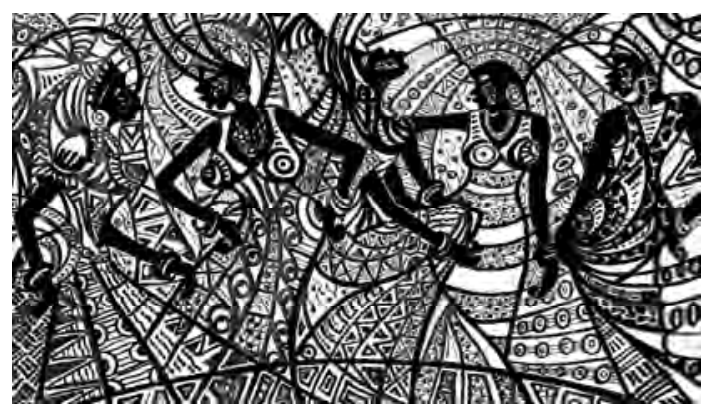

Fig. 1c: The final design for Mural 1

\section{Production of Textile Mural 1}

Textile techniques employed were batik, tieand-dye, appliqué, screen printing and embroidery. Step 1: Transfer of design

1. The A4 size paper design (Fig. 1c) was first enlarged to $229.5 \mathrm{~cm} \times 102.5 \mathrm{~cm}$ using the square enlargement method after which the enlarged design was transferred by means of carbon paper onto white mercerized cotton fabric measuring $274.5 \mathrm{~cm} \mathrm{x}$ $129.5 \mathrm{~cm}$.

2. The outlines of the design were traced out with 2B (soft) pencil to aid transfer of the design from paper to fabric. Identified sections of the design to be dyed yellow, orange, green and violet were indicated on the fabric with numbers to aid effective waxing and dyeing.

Step 2: Batik

1. A measured quantity of vat dye was dissolved in a bowl of warm water and poured into a sodium hydroxide and sodium hydrosulphite (reducing and swelling agents) solution in a large bowl. Separate dye liquors were prepared for each of the four colours of dye used - yellow, orange, green and violet.

2. Using a piece of cushion foam with a tapered edge as a drawing tool, molten wax was applied to all the white areas in the design to resist dye absorption. The waxed fabric was dyed yellow and dried. The waxing, dyeing and drying processes were repeated for the orange, green and violet sections of the design in that order. Figure 2 shows the dyed fabric prior to dewaxing.

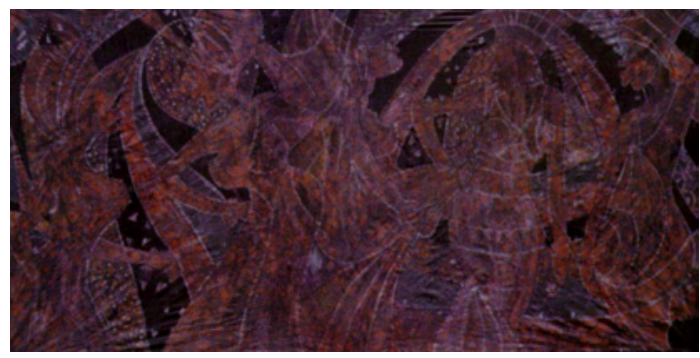

Fig. 2: The design after waxing and dyeing 
3. De-waxing involved immersing the dry waxed fabric in boiling water and gently swirling it around to melt the wax and expose the covered dye colours. When done, the fabric was removed, drained of excess water, washed in cold water, rinsed, dried and ironed to obtain the batik design in Fig. 3.
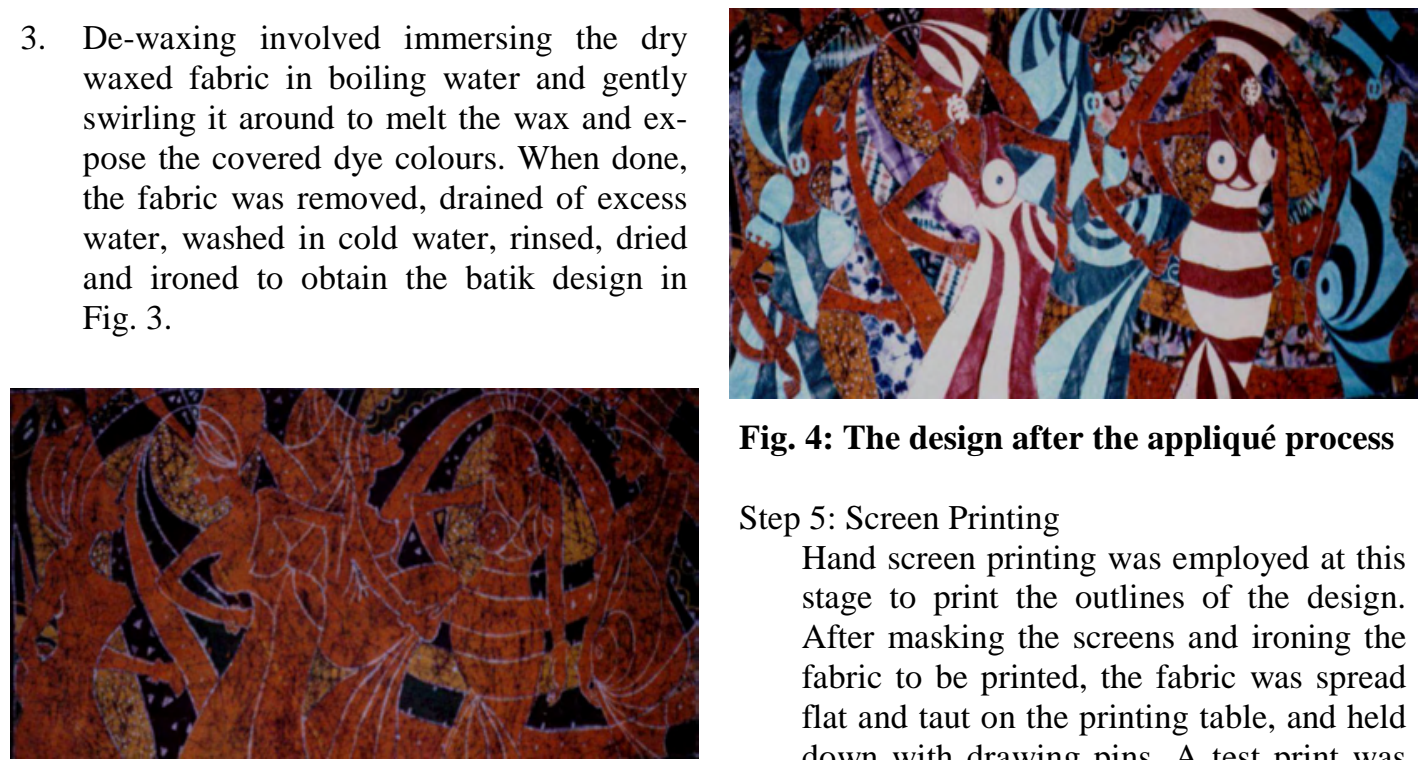

Fig. 4: The design after the appliqué process

Step 5: Screen Printing

Hand screen printing was employed at this stage to print the outlines of the design. After masking the screens and ironing the fabric to be printed, the fabric was spread flat and taut on the printing table, and held down with drawing pins. A test print was made with talcum powder to check the precision of repeat prints on the fabric before using the squeegee and printing paste to register the design on the screen onto the fabric.

Different samples of tie-dyed fabrics based on the marbling, splash, diagonal stripes, diamond-shapes and tritik (sewing) resist styles were produced and used for the appliqué aspect of the murals.

Step 4: Appliqué

1. Appliqué patterns were traced from the batik fabric (Fig. 3) onto the $274.5 \mathrm{~cm} \mathrm{x}$ $129.5 \mathrm{~cm}$ plain damask fabric, and previously created tie-dye fabrics.

2. The traced patterns on the fabrics were cut out with scissors, strengthened with fabric stiffener of the same size on its adhesive side by ironing it against the fabric on an ironing board. The cut out patterns were arranged and glued onto the batik design (Fig. 3) using PVA glue to give the appliqué effect.

3. Different sizes of seashells were glued or stitched to selected areas of the fabric to serve as necklaces for the figures and to enhance the aesthetic appeal of the design. The result after applying the appliqué technique is shown in Fig. 4.

\section{Step 6: Embroidery}

An electric embroidery machine was used to sew zigzag stitches of varying lengths and thickness along the edges of the appliqué patterns to secure them to the base fabric and also to create ornamental stitching effects in the mural. Figure 5 shows the finished and framed Textile Mural 1.

\section{Design concept}

Mural 1 is titled "The Pride of Ghanaian Women" to commemorate the colourful manner in which Ghanaian women adorn themselves. It is a polychromatic fabric mural depicting five female figures gorgeously dressed in contemporary Ghanaian costume. The figures have different postures and their widely opened eyes imply that the four women seem to have been startled by information being communicated by the woman on the left side of the design. The linked figures symbolize fraternity, unity, communality, love, support and rapport among the women. The colourfully patterned 


\section{Howard and Opoku-Asare}

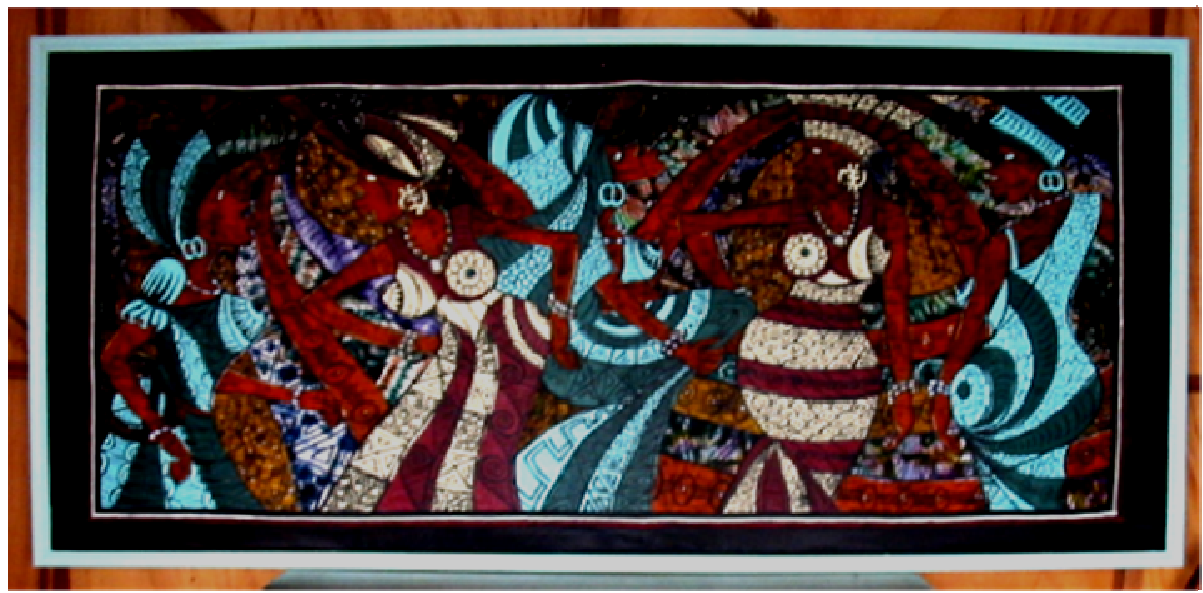

Fig. 5: Finished Textile Mural 1

(Displayed in Faculty of Art Gallery, KNUST)

adornment reflects the vivacious nature and dress sense of Ghanaian women, and their attraction to high quality fabrics with vibrant colours and symbolic patterns.

Significantly, women are the ones who usually sell and make women's clothing in Ghana. Cloth sellers and seamstresses are also known to popularize new fabrics and fashion accessories and so every opportunity to showcase and talk about what is in vogue counts. The market place, shopping centres and social gatherings are known to offer platforms for talk about fashionable fabrics and clothing styles. The theme reflects a scenario that is very common among Ghanaian women and how they project themselves in fashion clothes. The animated scene is characteristic of women's meetings and how animated they can get as they share ideas, make conversation and relate to each other on passionate topics. The lineal flow of garments is significant in terms of the line of communication, the essence of rapport and support that Ghanaian women enjoy from each other.

The flow of lines also projects the uniqueness of each figure's garment design, fabric colour, dress sense, headgear and posture against the background design. The mural design also portrays the unique ways in which the pride of owning a fashion article shows through posture, action and gestures. Anyone seeing this will have enough to see and think about or discuss with others, suggesting its viability as an effective tool for communicating critical information for public education. The actions and postures of the figures also depict "pride" in the sense that, psychologically, beautiful fabric or garment tends to boost the ego or personality of the wearer, making him or her proud and confident in public.

\section{Textile Mural 2: The KNUST Crest}

Mural 2 is based on the Kwame Nkrumah University of Science and Technology (KNUST) crest (see Fig. 6) with a printed Nyansapo wosane no badwemma (it takes high intellect to untie the wisdom knot) banner. The mural was done in the characteristic red, gold, green and black national colours to reflect the true representation of the KNUST crest. The aim of creating this mural was to project the image of this noble academic institution of higher learning in an innovative medium and to also prove the viability of fabric and composite textile techniques for making complex as well as simple murals of high aesthetic and educational values. 
The textiles techniques employed to produce this mural were appliqué, screen printing and embroidery respectively. The same procedure described for Mural 1 was employed for Mural 2.

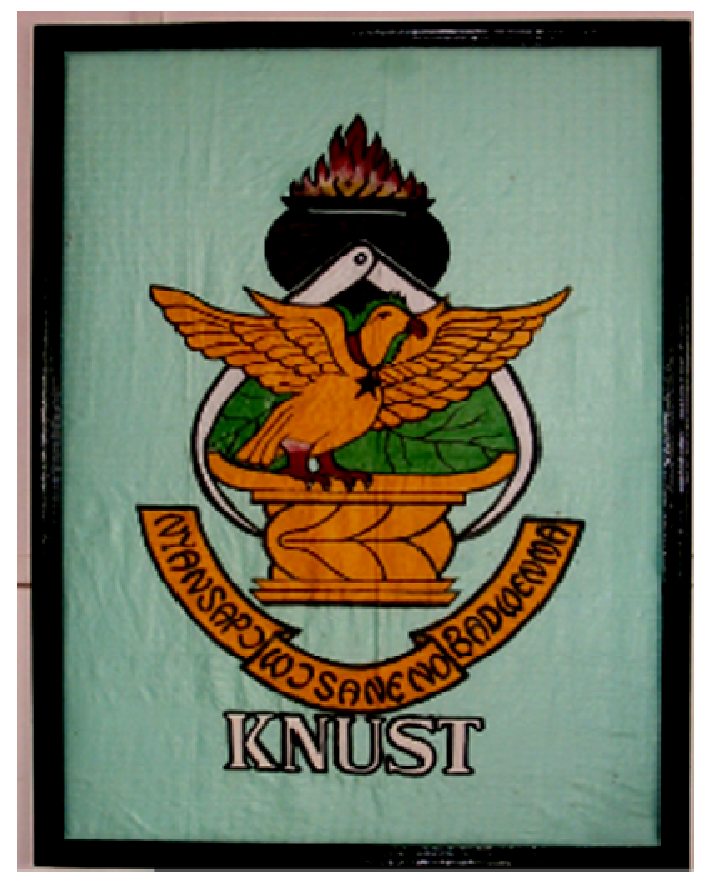

Figure 6: Finished Textile Mural 2

(Displayed in the office of the $2^{\text {nd }}$ researcher)

\section{CONCLUSION}

Although fabric is traditionally meant for clothing, the study has revealed extensive possibilities in using plain woven cotton fabric to produce murals that can be managed easily because dust can be brushed off them; they can also be folded into smaller sizes for easy transportation and repositioning. This new generation of murals can serve as the foundation for effective teaching and learning of Fabric Decoration Techniques, Elements and Principles of Art, Arts Appreciation, Aesthetics and Criticism, and Imaginative Composition within the Visual Arts curricula followed in secondary and tertiary art institutions in Ghana. Such murals can enhance the ambience of waiting environ- ments while also providing food for thought, emotional therapy, pleasure, focus and calmness to relieve nervousness, stress and anxiety in any hotel lobby, airport departure hall, banking hall, staff or students' common room, or out -patient-department of a health facility. The success of this experiment therefore opens a new avenue for muralists and textiles designers to explore other forms of pliable materials such as leather and polythene sheets to create murals to widen the scope of mural art for artistic development of Ghana.

\section{REFERENCES}

Adams, L. S. (1990). Art Across Time - Prehistoric to the Fourteenth Century. Volume 1 New York: McGraw-Hill, Inc.

Anaba, A. (1995). Symbolism in Murals of Nankam Speaking area of Upper East Region. MA Thesis, Kwame Nkrumah University of Science and Technology, Kumasi.

Asihene, E. V. (2004). A brief History of Art with special reference to West Africa. Woeli Publishing Services: Accra.

Bhuyan, R. (2011). Mural Painting Techniques. http://www.buzzle.com/articles/muralpainting-techniques.html. Retrieved 07/02/2012.

Campbell, B. (2003). Mexican Murals in times of Crisis. Tucson: University of Arizona Press.

Demoli, J. (2011). Mural Art-The Art of Painting Walls. http://murals.demolliart.com Retrieved 24/01/2012.

Donitz, R. J. (2004). A Guide to 1000 Los Angeles Murals. RJD Enterprises, Los Angeles. http//www.geocities.com/The tropics/6788/Murals.htm. Retrieved 24/10/2004.

Howard, E. K. (2006). “Design and Production 


\section{Howard and Opoku-Asare}

of a Mural using Woven Fabrics and Composite Textile Techniques". MA Thesis, Kwame Nkrumah University of Science and Technology, Kumasi.

Microsoft Encarta Standard (2009). Mural Painting. Retrieved 13/12/2010.

Osumi, T. (2000). A Mural Making Guide for K-12 Teachers. www.sscent.ucla.edu/aasc/ classweb/spring001/webmag - 197j/tony mur.html-33k. Retrieved 10/02/2004.
Scholastic (2012). Learning Through Wall Paintings. www.sscent-ucla/aasc/classweb/ s pring $001 /$ we b mag - $197 \mathrm{j} /$ t o n y murhtml/33k. Retrieved 08/02/2012.

Tarantino, A. (2011). Seattle Street Art - A Visual Time Capsule Beyond Graffiti. ht t p://en.wikipedia.org/wiki/ Mural\#Tile_mural. Retrieved 08/02/2012.

Wisegeek (2012). What is a mural? http:// www.wisegeek.com/what-is-a-mural.htm. Retrieved 24/01/2012. 